ARTICLE

\title{
Proximal and distal effects of genetic susceptibility to multiple sclerosis on the $\mathrm{T}$ cell epigenome
}

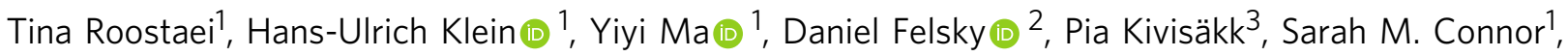
Alexandra Kroshilina ${ }^{1}$, Christina Yung ${ }^{1}$, Belinda J. Kaskow ${ }^{4}$, Xiaorong Shao ${ }^{5}$, Brooke Rhead ${ }^{5}$, José M. Ordovás (10 ${ }^{6}$, Devin M. Absher ${ }^{7}$, Donna K. Arnett (10 ${ }^{8}$, Jia Liu (i] ${ }^{9}$, Nikolaos Patsopoulos ${ }^{4}$, Lisa F. Barcellos ${ }^{5}$, Howard L. Weiner (ib ${ }^{4} \&$ Philip L. De Jager (i] ${ }^{1 凶}$

Identifying the effects of genetic variation on the epigenome in disease-relevant cell types can help advance our understanding of the first molecular contributions of genetic susceptibility to disease onset. Here, we establish a genome-wide map of DNA methylation quantitative trait loci in $\mathrm{CD}^{+}{ }^{+} \mathrm{T}$-cells isolated from multiple sclerosis patients. Utilizing this map in a colocalization analysis, we identify 19 loci where the same haplotype drives both multiple sclerosis susceptibility and local DNA methylation. We also identify two distant methylation effects of multiple sclerosis susceptibility loci: a chromosome 16 locus affects PRDM8 methylation (a chromosome 4 region not previously associated with multiple sclerosis), and the aggregate effect of multiple sclerosis-associated variants in the major histocompatibility complex influences DNA methylation near PRKCA (chromosome 17). Overall, we present a new resource for a key cell type in inflammatory disease research and uncover new gene targets for the study of predisposition to multiple sclerosis.

\footnotetext{
${ }^{1}$ Center for Translational and Computational Neuroimmunology, Department of Neurology and the Taub Institute for Research on Alzheimer's disease and the Aging brain, Columbia University Irving Medical Center, New York, NY, USA. ${ }^{2}$ Krembil Centre for Neuroinformatics, Centre for Addiction and Mental Health, University of Toronto, Toronto, ON, Canada. ${ }^{3}$ Alzheimer's Clinical and Translational Research Unit, Department of Neurology, Massachusetts General Hospital, Boston, MA, USA. ${ }^{4}$ Ann Romney Center for Neurologic Diseases, Brigham and Women's Hospital, Harvard Medical School, Boston, MA, USA. ${ }^{5}$ Genetic Epidemiology and Genomics Laboratory, University of California, Berkeley, CA, USA. ${ }^{6}$ Jean Mayer US Department of Agriculture Human Nutrition Research Center on Aging, Tufts University, Boston, MA, USA. ${ }^{7}$ HudsonAlpha Institute for Biotechnology, Huntsville, AL, USA. ${ }^{8}$ College of Public Health, University of Kentucky, Lexington, KY, USA. ${ }^{9}$ Advanced Science Research Center at the Graduate Center, Neuroscience Initiative, City University of New York, New York, NY, USA. 『email: pld2115@cumc.columbia.edu
} 
M ultiple sclerosis (MS) is a genetically complex inflammatory disease of the central nervous system. Despite a growing list of drugs that prevent relapses ${ }^{1}$, there is, as yet, no preventive strategy for MS, highlighting our limited understanding of the molecular events leading to disease onset. Given that genetic risk factors can be presumed to be causally linked to MS, they serve as a robust starting point for understanding the mechanisms predisposing to the disease. The most recent genome-wide association study (GWAS) of MS susceptibility ${ }^{2}$, provides compelling evidence for the effects of 32 independent susceptibility variants in the major histocompatibility complex (MHC) and 200 autosomal non-MHC susceptibility variants.

Our understanding of the functional consequences of these variants remains limited ${ }^{2-5}$. Here, we examined the first level of downstream molecular changes: alterations in the epigenome. Specifically, we focused on mapping methylation of CpG dinucleotides, an epigenomic mark for which nucleotide-resolution data can be reliably produced throughout the genome. Insights into the functional consequences of risk alleles on the epigenome can, in turn, be used to further elucidate the causal chain of biological mechanisms involved in disease onset. While the effects of many variants are shared, others demonstrate cell-type and context specificity. As $\mathrm{CD} 4^{+} \mathrm{T}$ cells are believed to play a major role in the pathogenesis of MS and other inflammatory disorders ${ }^{3}$, we purified these cells from MS patients to study the epigenome in a disease-relevant context.

$\mathrm{CD} 4^{+} \mathrm{T}$ cells have been interrogated in a number of recent quantitative trait locus (QTL) studies, albeit mainly from a gene expression (eQTL) perspective and mostly in healthy controls ${ }^{3,6}$. Here, using the Illumina MethylationEPIC array, we generated genome-wide DNA methylation profiles from $\mathrm{CD} 4^{+} \mathrm{T}$ cells isolated from 156 MS patients. These data were used to generate a resource outlining the genome-wide genetic architecture of $\mathrm{T}$ cell DNA methylation levels in a disease state. We then performed a comprehensive set of analyses to determine the effects of MS risk loci. As a result, we (1) provide a genome-wide cis- DNA methylation QTL (mQTL) map of $\mathrm{CD}^{+}{ }^{+} \mathrm{T}$ cells in MS patients, which can be utilized in future studies of MS and other inflammatory diseases, (2) identify cis- effects of MS genetic susceptibility variants on nearby $\mathrm{CpG}$ dinucleotide methylation, (3) discover and validate a trans-mQTL effect of an MS variant, and (4) demonstrate that polygenic scores of MS susceptibility influence DNA methylation at specific CpG dinucleotides and suggest the convergence of the effects of multiple variants on methylation levels in distal $\mathrm{CpG}$ sites.

\section{Results}

Data generation. We selected subjects from participants in the Comprehensive Longitudinal Investigation of Multiple Sclerosis at the Brigham and Women's Hospital (CLIMB) study ${ }^{7}$ that fulfilled our selection criteria: (1) age 18-55 years old, (2) a diagnosis of MS fulfilling 2010 McDonald criteria, (3) a relapsingremitting disease course at the time of sampling, (4) being on one of two disease-modifying therapies (either glatiramer acetate [GA] or dimethyl fumarate [DMF]) at the time of sampling, (5) no evidence of disease activity in the prior 6 months, (6) no steroid use in the preceding 30 days, and (7) an Expanded Disability Status Scale (EDSS) score between 0 and 4. A prospectively collected, cryopreserved vial of peripheral blood mononuclear cells (PBMC) was accessed for each patient, and $\mathrm{CD} 4^{+} \mathrm{T}$ cells were purified after thawing using a positive selection strategy and a magnetic bead-based approach ("Methods"). DNA methylation profiles were generated using the DNA extracted from the purified $\mathrm{CD} 4^{+} \mathrm{T}$ cells using the Illumina MethylationEPIC array. We performed the mapping of $\mathrm{mQTL}$ effects using data from the 156 subjects for which both genotype and DNA methylation data passed all quality control measures, while adjusting for the effects of confounding factors including age, sex, and treatment (see "Methods"; demographic details are available in Table 1).

Genome-wide mapping of cis-mQTLs in primary $\mathrm{CD}^{+} \mathrm{T}$ cells of MS patients. From the 769,699 DNA methylation sites that passed quality control measures, we found evidence for the influence of cis genetic effects (within $\pm 1 \mathrm{Mb}$ of each CpG site) on the methylation levels of 107,922 CpGs (FDR-adjusted $p<0.05$ ) (Supplementary Data 1), after adjusting for the effect of technical and confounding variables ("Methods"). The high percentage of mCpGs (CpG sites which methylation levels are influenced by $\mathrm{mQTL}=14 \%)$ is comparable to findings from previous mQTL studies $^{8,9}$. As expected, the majority of lead cis-mQTL SNPs (mSNPs) are in a few kilobase distance from their target $\mathrm{CpGs}^{8}$, with $50 \%$ of mSNPs within $8 \mathrm{~kb}$ and $90 \%$ within $123 \mathrm{~kb}$ of their respective CpG sites (Supplementary Fig. 1). To examine enrichment of cis-mCpGs in specific functional regions of the genome, we performed enrichment analyses using chromatin state annotations modeled for $\mathrm{CD}^{+} \mathrm{T}$ cells (sample \#E043, Roadmap Epigenomics Project ${ }^{10}$ ), in addition to annotations for $\mathrm{CpG}$ islands and gene transcribed regions. We found lower than expected number of $\mathrm{mCpGs}$ in transcription start sites (TSS), transcribed regions, and CpG islands (which often occur at or near TSS). In contrast, enrichment was observed for $\mathrm{mCpGs}$ in flanking areas to TSS, flanking areas to CpG islands (island shores), and enhancer regions (Fig. 1a). Subset analysis revealed that the enrichment patterns were largely similar between the two versions of the Infinium methylation profiling platform: HumanMethylation 450 and MethylationEPIC (Supplementary Fig. 2a). The identified mCpGs were distributed over 59,603 unique functional chromatin segments, $45 \%$ of which $(n=26,987)$ were not covered by HumanMethylation450 array (Supplementary Fig. 2b), highlighting the added value of using MethylationEPIC over the 450 array in our study.

We then compared our results with findings from a previous cis-mQTL study of naïve $\mathrm{CD} 4^{+} \mathrm{T}$ cells from 132 healthy controls performed as part of the BLUEPRINT Epigenome Project ${ }^{9}$. Of note, the Illumina $450 \mathrm{~K}$ array used for measuring DNA methylation in the BLUEPRINT study contains only one-half of the number of $\mathrm{CpGs}$ tested in our study. Hence, our comparison is limited to the subset of CpGs shared between the two arrays. Of our 107,922 identified mCpG-mSNP pairs, 40,853 (38\%) were tested in the BLUEPRPINT study. Of those, 38,776 (95\%) showed the same direction of effect, 33,128 (81\%) also had a nominally significant $p$ value $(p<0.05)$, and $25,544(63 \%)$ had an FDRadjusted $p<0.05$ in the BLUEPRINT data (Fig. 1b, Supplementary Data 1). The agreement between the two studies is further visualized using the estimated standardized regression coefficients for the effects of SNPs on the methylation of target CpGs (Fig. 1b).

In order to investigate whether the $5 \%$ of mQTL effects that showed opposite direction of effect in the two datasets are MS- or treatment-specific, we performed gene set enrichment analyses on the annotated genes for the relevant mCpGs. No enrichment was observed for MS-susceptibility associated genes ${ }^{2}$. Moreover, these $\mathrm{mCpGs}$ were not enriched for differentially-methylated CpGs in $\mathrm{CD}^{+} \mathrm{T}$ cells of DMF-treated MS-patients ${ }^{11}$. Further comparison with the subset of available cis-mQTL results $(n=18,221 \mathrm{mCpG}-$ mSNP pairs) from a study of $\mathrm{CD}^{+} \mathrm{T}$ cells from 717 related individuals of the GOLDN study (dbGaP Study Accession: phs000741.v2.p1) (Supplementary Data 1) confirmed that the majority of the identified cis-mQTLs had a similar direction of 


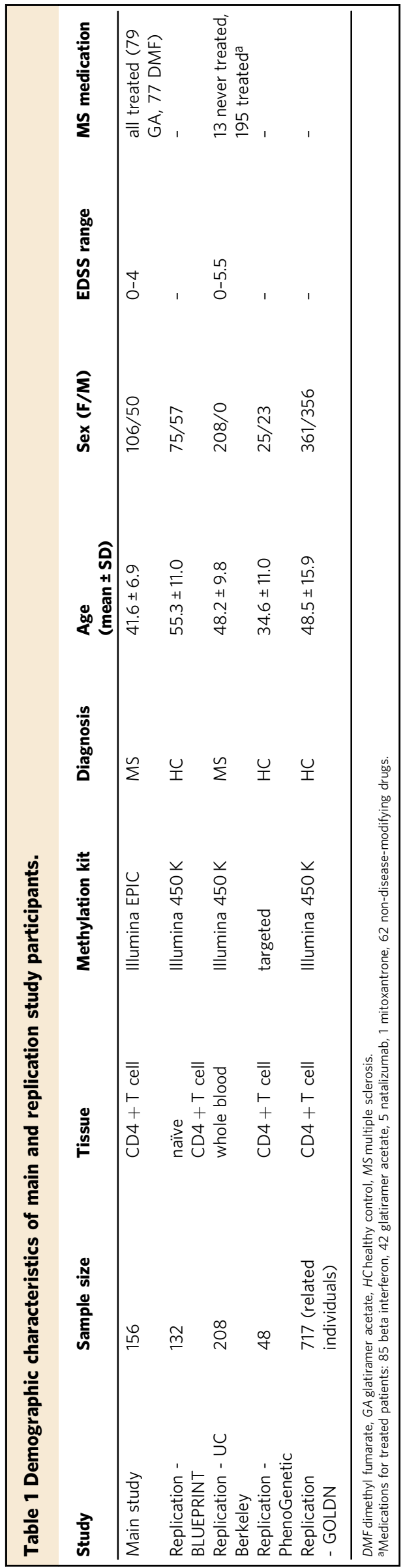

effect in all three datasets (92.4\%) (Supplementary Fig. 3). The slight differences between our results and the two other studies might have arisen from technical variation and populationspecific effects, as well as the choice of cell type between our study and the BLUEPRPINT study $\left(\mathrm{CD} 4^{+} \mathrm{T}\right.$ cells comprising both naïve and memory cells in our sample vs. naïve-only $\mathrm{CD} 4^{+}$ $\mathrm{T}$ cells in the BLUEPRPINT study) and disease status (MS patients in ours vs. healthy controls in the BLUEPRINT and GOLDN studies). Nonetheless, the high degree of agreement between the three studies provides validation for our methylomewide results.

Colocalized cis-mQTL effects of non-MHC MS susceptibility loci. Of the 107,922 significant cis-mQTL effects, lead mQTL SNPs for $3090 \mathrm{CpGs}$ were located within $100 \mathrm{~kb}$ of the lead nonMHC MS-associated $\operatorname{SNPs}^{2}(n=200)$. To determine the loci with likely shared causal effects on cis DNA methylation and MS susceptibility while minimizing false positive findings as a consequence of coincidental overlap because of linkage disequilibrium (LD), we performed Bayesian colocalization analyses $^{12}$ between MS and cis-mQTL effects. MS susceptibility summary association statistics were taken from the discovery phase of the recent MS GWAS ${ }^{2}$ performed using data from 41,505 individuals. We found strong evidence of colocalization (posterior probability $>0.8$ ) between $19 \mathrm{MS}$-associated loci and 43 cis-mQTL effects (Supplementary Data 2). The top three colocalized loci (posterior probability $>0.95$ ) are shown in Fig. 2a: the rs2248137 MS effect on chromosome 20 was colocalized with the cis-mQTL effect for cg14595058 upstream of the CYP24A1 gene; the rs1077667 MS effect on chromosome 19 was colocalized with the cis-mQTL effect for cg23071186 located in the TNFSF14 gene; and the rs7731626 MS effect on chromosome 5 was colocalized with cis-mQTL effects for $13 \mathrm{CpGs}$ located in and around the ANKRD55 and IL6ST genes (8 CpGs with posterior probability $>0.95$ and 5 CpGs with posterior probability $>0.8$ ) (CpG locations are illustrated in higher resolution in Supplementary Fig. 4). Further examination of the $13 \mathrm{CpGs}$ in the chromosome 5 colocalized locus revealed that they were all located in methylation open sea regions spanning over an area up to $172 \mathrm{~kb}$ from the lead MS SNP. Reference chromatin state annotation (Roadmap Epigenomics Project ${ }^{10}$ ) suggested that they were located in putative enhancer regions, and their methylation levels were positively correlated with each other (Supplementary Fig. 5). Colocalization with mQTL effects has previously been reported for this locus in the BLUEPRINT study ${ }^{9}$. The 16 other colocalized MS loci with posterior probability $>0.8$ and $<0.95$ affected methylation levels of $28 \mathrm{CpGs}$ including CpGs in the vicinity of C1ORF106, RGS14, AHI1, CHST12, ZNF767P, TRIM14, SLC15A3, RMI2, TBX6, TEAD2, CCDC155, NCOA5, and NCF4 genes (Supplementary Data 2).

We further compared the results of our colocalization analysis with the outcome of a similar analysis that we performed using cis-mQTL summary statistics obtained from the BLUEPRINT study. Data from 1,414 CpGs (out of 3,090) were shared between the two MS-cis-mQTL colocalization analyses, and high correlation was observed between colocalization posterior probability estimates (Spearman's $\rho=0.71$ ) (Fig. 2b). Of the 43 CpGs with colocalized MS-cis-mQTL effects in our data, eight were available in BLUEPRINT. All, except for the cg14130459 cis-mQTL effect in the SLC15A3 gene, showed replication, with comparable evidence of colocalization using the BLUEPRINT data (posterior probability estimates $>0.67$, Supplementary Data 2, Fig. $2 \mathrm{~b}$ ). The lack of colocalization evidence between MS and the cg14130459 cis-mQTL effect might be explained by the weaker mQTL association observed for cg14130459 in naïve $\mathrm{CD}^{+} \mathrm{T}$ cells of 
a cis-mCpGs enrichment for methylation, gene and chromatin features

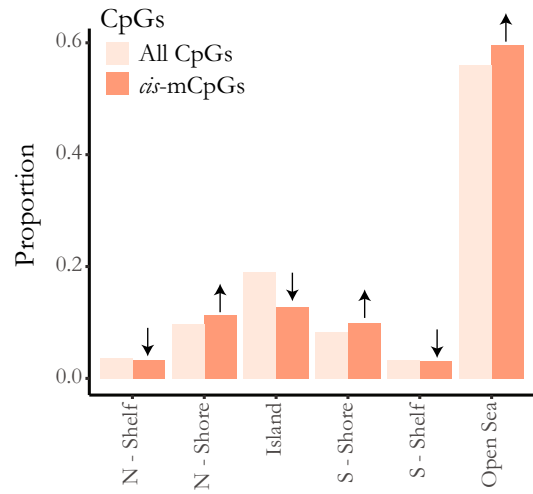

Relation to CpG island

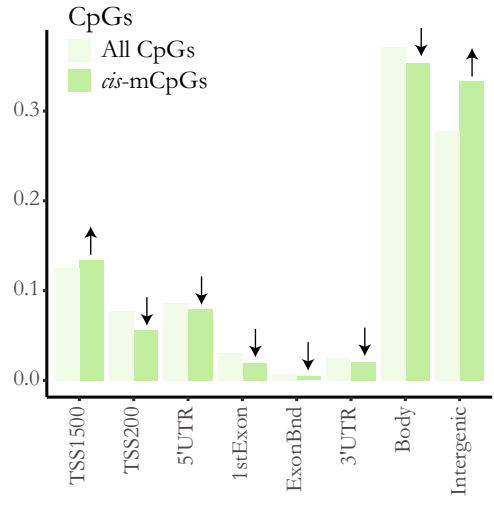

UCSC RefGene group

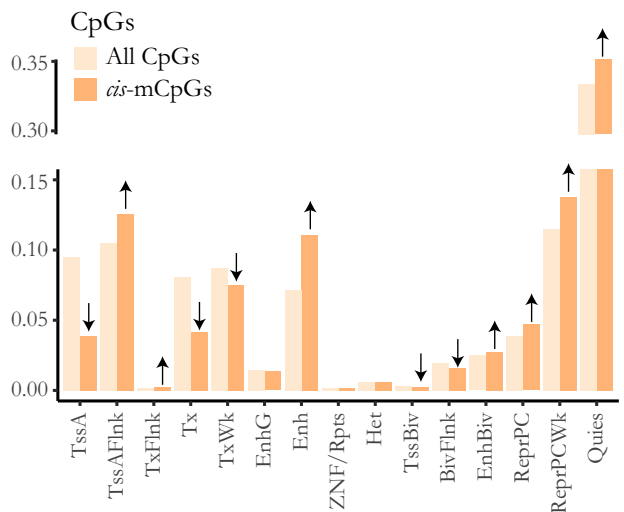

Chromatin states

b Comparison with cis-mQTL results from the BLUEPRINT study (naïve CD4 ${ }^{+} \mathrm{T}$ cells)
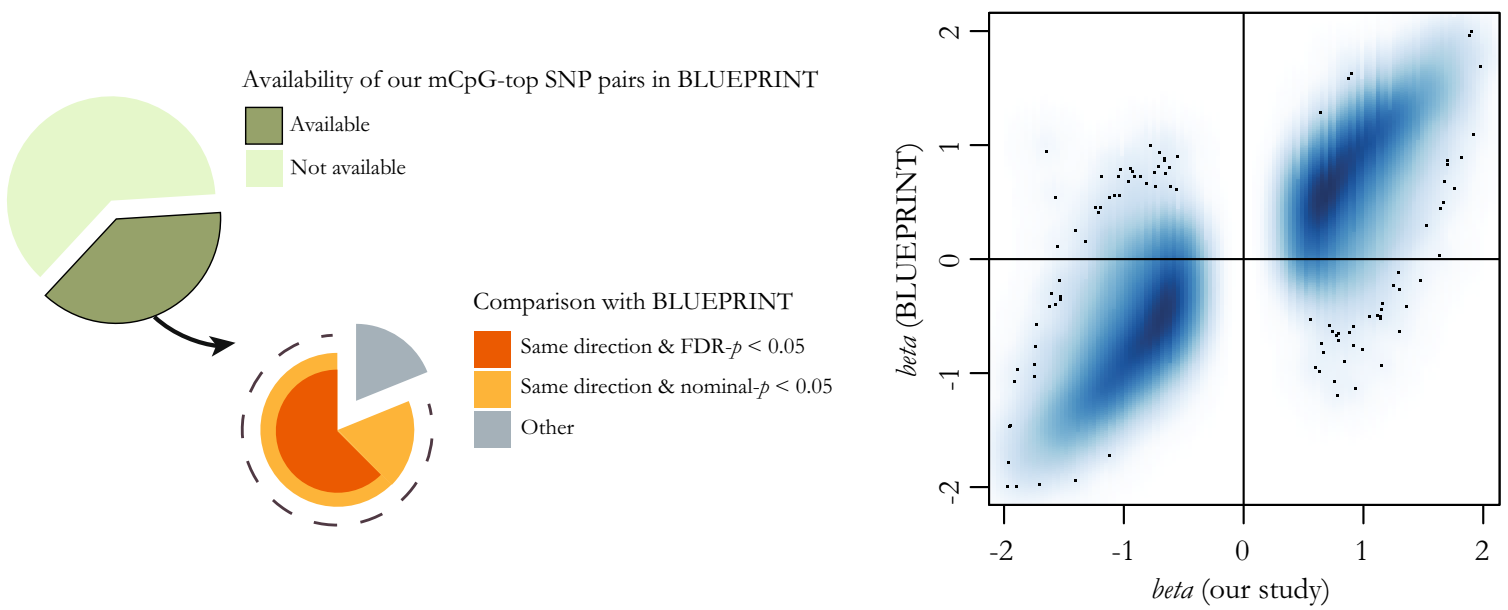

Fig. 1 Characterization of genome-wide significant cis-mCpGs and cis-mQTLs. a Enrichment of the identified cis-mCpGs in comparison to all tested CpGs in relation to UCSC CpG islands and gene functional regions (annotations from R IlluminaHumanMethylationEPICanno.ilm10b2.hg19 package) and chromatin states modeled for CD4 ${ }^{+}$T cells (annotations from Roadmap Epigenomics Project, sample \#E043). Significant enrichment/depletion are shown using upwards/downwards arrows, respectively. b Percentage of our identified cis-mCpGs available in the BLUEPRINT study and the comparison between the two studies regarding the direction of effect and significance of association for the lead mSNPs are shown on the left. Comparison between the effect sizes found in our study and in BLUEPRINT are shown on the right.

healthy participants ( $p=0.006$, BLUEPRINT study) in comparison to CD4 $4^{+}$T cells of MS patients ( $p=2.68 \times 10^{-10}$, our study).

Taking advantage of available RNA sequencing data from primary naïve and memory $\mathrm{CD} 4^{+} \mathrm{T}$ cells from a subset of our subjects $(n=36)$, we examined the gene expression correlates of the 43 identified CpGs with colocalized MS susceptibility and cismQTL effects. Our association analyses between methylation levels of the identified CpGs and expression levels of the genes in cis ( $\pm 1 \mathrm{Mb}$ of each $\mathrm{CpG}$ ) revealed significant DNA methylationgene expression associations at one of the colocalized effects (inverse correlation with ANKRD55 gene expression at 5\% FDR, top association: cg21124310: $p=2.7 \times 10^{-6}$ in naive and $p=1.2 \times 10^{-5}$ in memory $\mathrm{CD} 4^{+} \mathrm{T}$ cells). The MS risk allele at this locus, rs $7731626^{\mathrm{G}}$, has previously been linked with higher ANKRD55 expression, and the known cis-eQTL effect has been demonstrated to colocalize with the MS effect in $\mathrm{CD} 4^{+} \mathrm{T}$ cells of healthy individuals ${ }^{5,9}$. Similarly, we observed cis-eQTL effects for the MS SNP on ANKRD55 expression in both naive $\left(p=6.9 \times 10^{-4}, t=3.7\right.$, Fig. $\left.2 \mathrm{c}\right)$ and memory $\mathrm{CD} 4^{+} \mathrm{T}$ cells $\left(p=1.0 \times 10^{-4}, t=4.4\right)$ in our MS subjects. Our analyses further suggested that the effect of $\mathrm{rs} 7731626^{\mathrm{G}}$ on ANKRD55 expression is mediated by its effect on cg21124310 methylation ( $p$ for mediated effect $<2 \times 10^{-16}$ and $p$ for direct effect $>0.05$ in both naive and memory $\mathrm{CD} 4^{+} \mathrm{T}$ cells), while the effect of rs $7731626^{\mathrm{G}}$ on cg21124310 methylation is not mediated by its effect on ANKRD55 expression ( $p$ for mediated effect >0.05) (see "Methods"). We note that our sample size for the DNA methylation-gene expression sub-analysis was small, limiting our power to identify the weaker gene expression correlates of other colocalized MS-mQTL effects. However, the identified genetic loci and their corresponding CpGs can be used in future studies of proper sample size with specific focus on identifying the gene expression correlates of these loci and their relationship with methylation effects.

We also reviewed the cis-eQTL studies of peripheral blood mononuclear cells $s^{2}$, purified blood immune cells $s^{3,6,9}$, and brain tissue $^{8}$ from MS patients or healthy controls, and summarized the results for lead MS and lead mQTL SNPs of the colocalized MScis-mQTL effects in Supplementary Data 3. We note that 3 of the colocalized loci (SNPs in the vicinity of C1ORF106, CMC1, and 
a
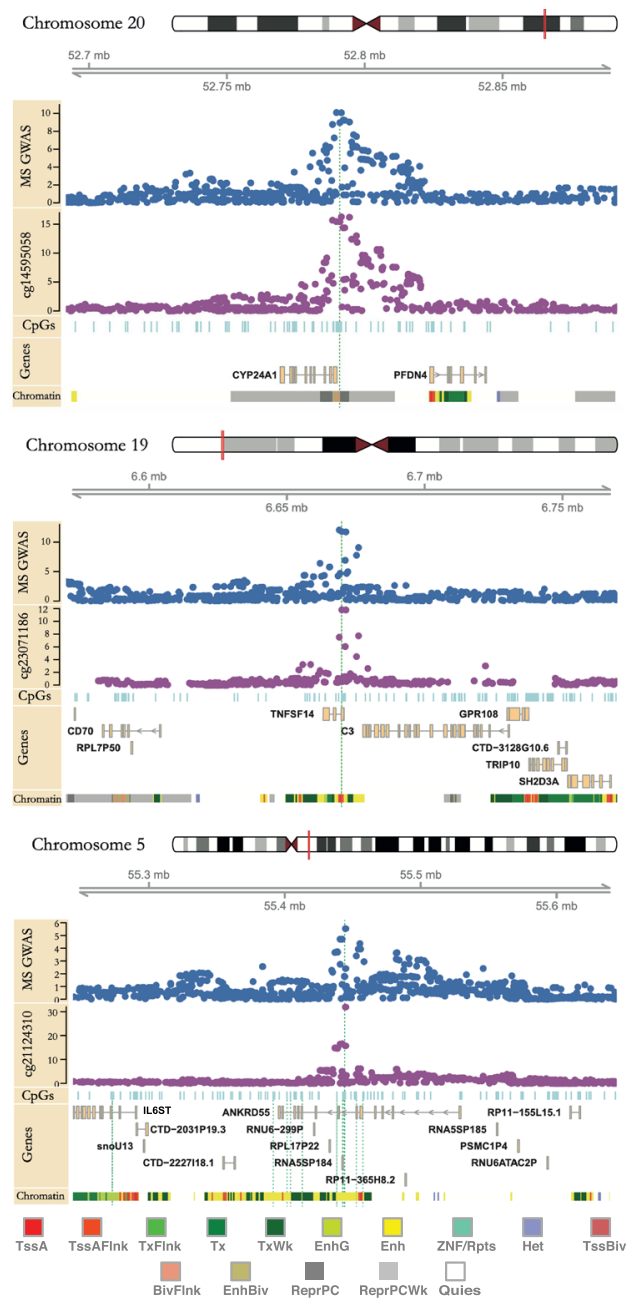

b

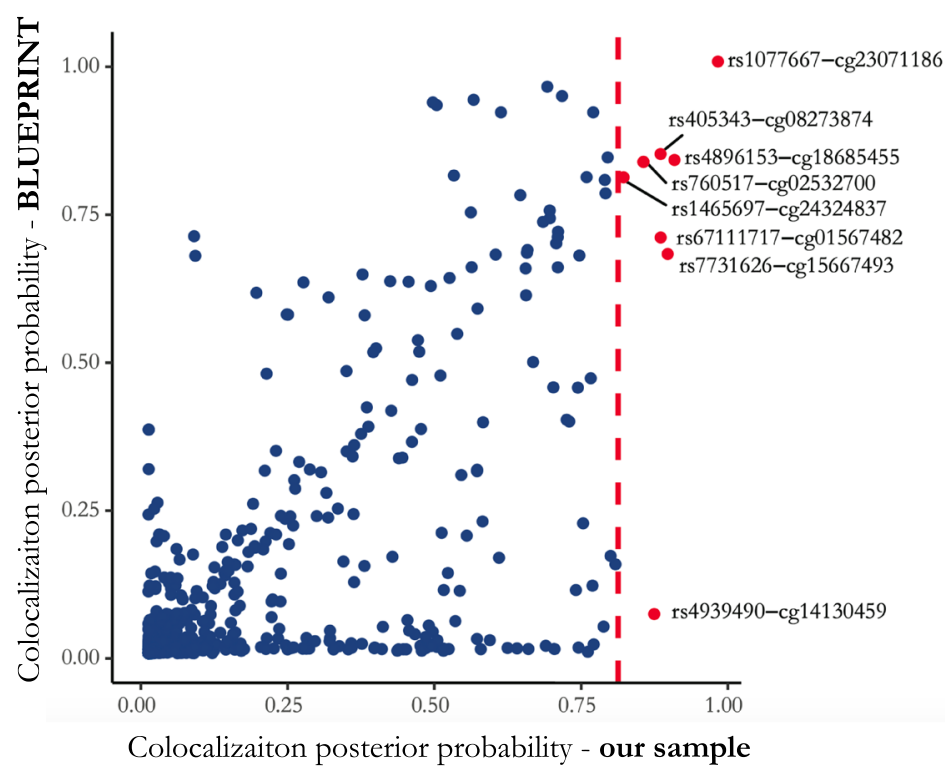

C
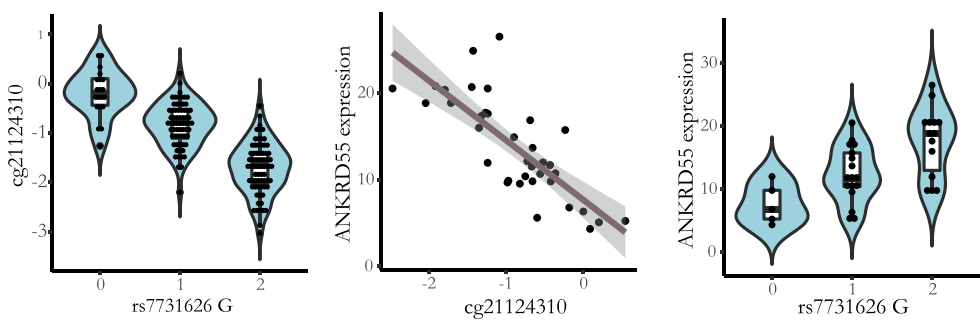

Fig. 2 Colocalized cis-mQTL effects of MS susceptibility loci. a The top 3 colocalized MS-cis-mQTL effects are illustrated (posterior probability >0.95 for colocalization between MS susceptibility and $\geq 1$ cis-mQTL effect). The MS GWAS rows (blue dots) show -log( $p$-value) of association between SNPs and MS susceptibility in the discovery phase of the 2019 IMSGC GWAS. CpG rows (plum dots) show -log(p-value) of association between SNPs and the specified cis-CpGs methylation levels. Locations of the local CpGs measured with the Infinium MethylationEPIC kit are shown using light blue vertical lines. Gene exon/intron positions are based on Ensembl 93. Chromatin state annotations for CD4+ T cells are downloaded from the Roadmap Epigenomics Project (sample \#E043). Green vertical lines represent the genomic location of the colocalized CpGs: Long vertical lines traversing all rows represent the top specified CpGs, while other vertical lines represent the additional colocalized cis-mCpGs with posterior probability $>0.8$. All genomic positions are in GRCh37 (hg19) coordinates. b Comparison between MS-cis-mQTL colocalization posterior probabilities using mQTL summary statistics from our study and BLUEPRINT for the available common CpGs. Vertical red line represents the threshold for high colocalization posterior probability in our study (i.e., 0.8). c The cis-mQTL, cis-eQTL, and CpG-mRNA association for the chromosome 5 top MS-cis-mQTL colocalized effect ( $n=156,36$ and 36 , respectively). Methylation levels are shown in M-values. Gene expression values are in TPM. Boxplot center lines represent median; the lower and upper hinges correspond to the 25th and 75th percentiles; the lower/upper whisker extends from the hinge to the smallest/largest value no further than $1.5 \times$ interquartile range. Error band represents $95 \%$ confidence interval.

SOX8 genes) have not been previously associated with cis-eQTL effects in any cell types in the above-mentioned studies, and five additional loci (SNPs close to EIF3B, TMEM25, TNFSF14, CD40, and CYP24A1 genes) have been associated with cis-eQTL effects in cell types other than $\mathrm{CD} 4^{+} \mathrm{T}$ cells. This information highlights the need for further investigation of the functional effects of these loci.

Colocalized trans-mQTL effects of non-MHC MS susceptibility loci. To investigate the influence of MS susceptibility loci on DNA methylation at distant CpG sites (trans-mQTL effects), we first mapped the trans-mQTL effects of all variants within $100 \mathrm{~kb}$ of the lead MS-associated SNPs. We then performed colocalization analysis and found evidence for colocalization (posterior probability $>0.95)$ of the effect of the MS locus centered on rs3809627 (chromosome 16) and three trans-mQTL effects. All three trans-mCpGs were located in a CpG island on chromosome 4 in the area flanking a transcription start site in the PRDM8 gene ( $p$-value for the top colocalized mQTL effect: rs3809627$\operatorname{cg} 27018912=5.7 \times 10^{-11}, t=7.1$, colocalization posterior probability $=0.98$ ) (Fig. 3a, Supplementary Fig. 6, Supplementary Data 2). While this MS locus centered on rs3809627 also showed colocalization with 2 cis-mQTL effects (CpGs in an enhancer region in the TBX6 gene; colocalization posterior probability $=$ 0.92), the methylation levels of the trans and cis mCpGs were not correlated (Supplementary Fig. 7), suggesting that the transmQTL effects of rs3809627 were not directly mediated through its cis-mQTL effects. 
$\mathbf{a}$
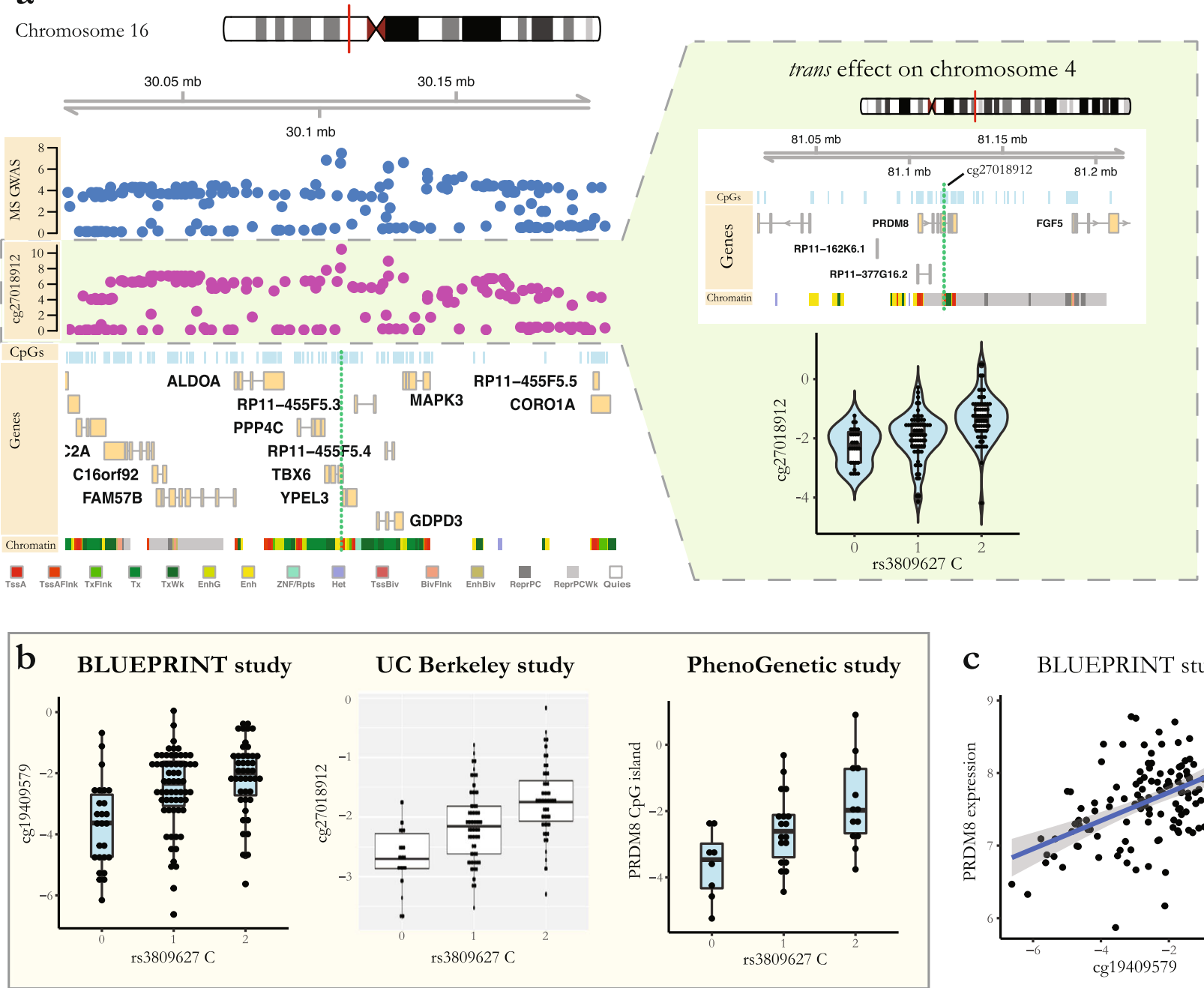

C BLUEPRINT study

Fig. 3 Colocalized trans-mQTL effect of MS susceptibility locus centered on rs3809627. a The top colocalized MS-trans-mQTL effect observed between MS susceptibility locus centered on rs3809627 in chromosome 16 and cg27018912 located in a CpG island in chromosome 4. Left: Association (-log(pvalue)) between SNPs located in the chromosome 16 locus and MS susceptibility (blue dots) and the trans-mCpG cg27018912 methylation levels (plum dots), accompanied by visualization of the $\mathrm{CpGs}$, genes and modeled chromatin states in the chromosome 16 locus. Green vertical lines represent additional colocalized cis-mCpGs (posterior probability >0.8). Right: Genomic position of the trans-mCpGs (cg27018912, cg19409579 and cg04235768) on chromosome 4 (vertical green lines) in relation to nearby CpGs, genes, and modeled chromatin states. The top colocalized trans-mQTL effect is shown in the bottom using methylation M-values $(n=156)$. b Replication of the identified trans-mQTL effect between rs3809627 MS susceptibility variant (chromosome 16) and CpGs in the CpG island located on chromosome 4 in three independent studies. Methylation levels are shown in M-values. Data is shown for cg19409579 in the BLUEPRINT study as the top available trans-mCpG measured with the Infinium HumanMethylation450 array. Methylation data shown for the PhenoGenetic study are the average methylation levels of all the measured CpGs in the identified CpG island. Boxplot center lines represent median; box limits represent upper and lower quartiles. c Significant CpG-mRNA association was observed between cg19409579 trans-mCpG and PRDM8 gene expression in the BLUEPRINT study. Methylation levels are in M-values. Gene expression levels are ComBat normalized values available from the BLUEPRINT. Error band represents $95 \%$ confidence interval. Boxplots (a, b): center lines represent median; the lower and upper hinges correspond to the 25th and 75th percentiles; the lower/upper whisker extends from the hinge to the smallest/largest value no further than $1.5 \times$ interquartile range.

We replicated our trans-mQTL finding - rs3809627 on chromosome 16 and the chromosome 4 CpG island-in three independent datasets (Fig. 3b). First, we found the same effect using the BLUEPRINT naïve $\mathrm{CD} 4^{+} \mathrm{T}$ cell DNA methylation data $\left(p=4.8 \times 10^{-6}\right)$. Second, we replicated the trans-mQTL in a dataset of DNA methylation profiles from whole blood of 208 MS patients from UC Berkeley $\left(p=5.4 \times 10^{-13}\right)$. Third, we analyzed targeted methylation data from $\mathrm{CD}^{+} \mathrm{T}$ cells purified from 48 healthy participants in the PhenoGenetic study ${ }^{13}\left(p=7.1 \times 10^{-4}\right)$ (see "Methods" for more details). These results generate high confidence in the trans-mQTL effect of rs3809627 and establish it as an effect seen in both MS and healthy subjects. Given the previous implications of activated $\mathrm{CD} 4^{+} \mathrm{T}$ cells in the pathophysiology of MS, we had generated, in parallel, a dataset of ex vivo activated $\mathrm{CD} 4^{+} \mathrm{T}$ cells using a subset of the PhenoGenetic subjects $(n=28)$ to investigate the effect of ex vivo activation on the methylation of CpGs of interest. We did not observe a significant difference in the CpG island methylation in the pairs of nonstimulated and stimulated samples $(p=0.95)$. However, assessing the functional effects of the trans-mCpGs using the BLUEPRINT data, we observed a highly significant positive association between cg19409579 methylation level and PRDM8 mRNA expression in naïve $\mathrm{CD} 4^{+} \mathrm{T}$ cells $(n=127, \rho=0.49$, association $p$ value adjusted for age and sex $=4.7 \times 10^{-9}$, Fig. $3 \mathrm{c}$ ). 

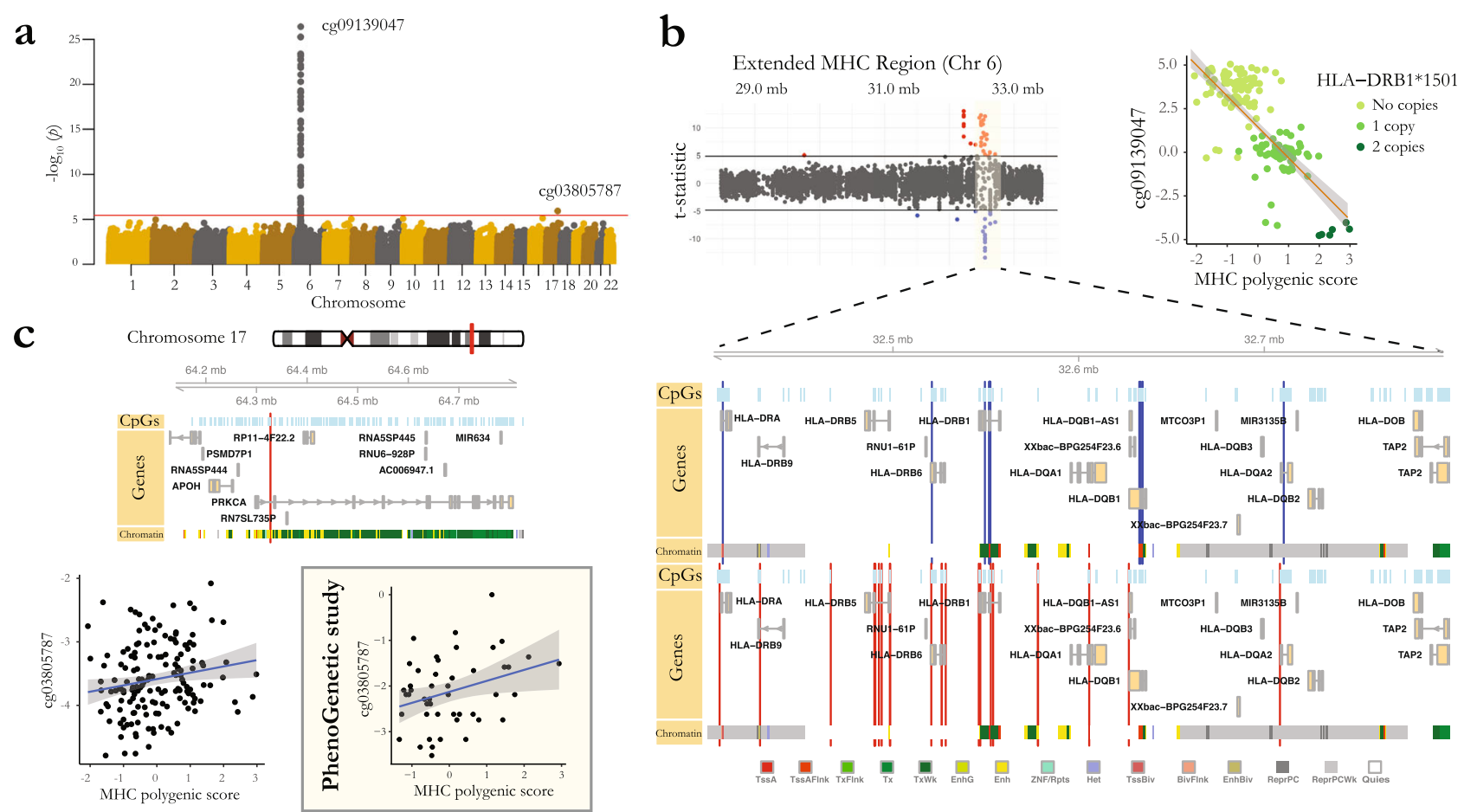

Fig. 4 cis and trans DNA methylation effects of MS MHC polygenic score. a Manhattan plot shows association $p$-values between CpG methylation levels and MS-associated MHC polygenic score. Red horizontal line shows the significance threshold (FDR $<5 \%$ ). Top CpGs in the two significant regions are specified. $\mathbf{b}$ t-statistics for positive and negative associations between the methylation levels of the $\mathrm{CpGs}$ located inside the extended MHC region and the MHC polygenic score are shown (red: positive, blue: negative). The inset illustrates the genomic location of the significant $\mathrm{CpGs}$ in the MHC class II region in relation to nearby $\mathrm{CpGs}$, genes, and modeled chromatin states. Blue/red vertical lines represent the locations for $\mathrm{CpGs}$ with negative/positive associations with the MHC polygenic score, respectively. Association between the top identified CpG, cg09139047 located in the HLA-DRB1 gene, and MHC polygenic score (shown in Z-scores) is visualized in the top right corner. Colors represent the number of HLA-DRB1*1501 alleles. c The genomic location for cg03805787 (associated with the MHC polygenic score in chromosome 17, red vertical line) is shown in relation to surrounding CpGs, genes, and modeled chromatin states. Bottom panel illustrates the association between cg03805787 methylation levels (M-values) and MHC polygenic score ( $Z$ scores) in our main study of MS subjects, as well as the replication in the healthy subjects of the PhenoGenetic study. Error bands (b, c) represent $95 \%$ confidence interval. $\mathrm{MHC}=$ Major Histocompatibility Complex.

cis and trans DNA methylation effects of an MS susceptibility polygenic score for the MHC. The MHC has a complex longrange LD structure and harbors 32 independent genome-wide significant associations with $\mathrm{MS}^{2}$, including the haplotype with the largest MS-associated risk, HLA-DRB1*1501. Given the region's complexity, we elected to focus our investigation on an assessment of the summary risk of MS based on the susceptibility variants found within the MHC. Specifically, we calculated a weighted MHC polygenic score representing the aggregate genetic effects of all of the independent susceptibility variants in this region and used this measure as the outcome for our methylomewide association study (MWAS). Our MWAS revealed significant associations between the MS MHC polygenic score and the methylation of $55 \mathrm{CpGs}(\mathrm{FDR}<0.05)$, 54 of which were located inside the extended MHC region on chromosome 6, and one was located on chromosome 17 (Fig. 4a, Supplementary Data 4, Supplementary Fig. 8).

The 54 identified CpGs located in the MHC region consisted of both hypo- and hyper-methylated CpGs in association with a higher MS MHC polygenic score. The majority of these CpGs were located in the MHC class II region, which also included the most significant association (a CPG located in the HLA-DRB1 gene) (Fig. 4b, Supplementary Fig. 9). The majority of the hypomethylated CpGs were located in two $\mathrm{CpG}$ islands in or around the transcription start sites of HLA-DRB1 and HLA-DQB1 genes, although both genes also had a few hyper-methylated CpGs. On the other hand, the majority of the hyper-methylated CpGs were located in HLA-DRB5, HLA-DRB6 and LOC101929163 (XXbacBPG154L12.4) (Supplementary Data 4, Supplementary Fig. 10). Our findings are supported by previous studies ${ }^{14,15}$ which also observed hypo- and hyper-methylated CpGs in the MHC class II region in MS patients in comparison to healthy controls and also in association with the HLA-DRB1*1501 genotype. Moreover, replication analysis using the BLUEPRINT data showed compelling evidence of association with the MHC polygenic score for all available CpGs (Supplementary Data 4).

The only CpG significantly associated with the MS MHC polygenic score outside of the MHC region was located in an enhancer of the PRKCA gene on chromosome 17 (cg03805787, $p=1.2 \times 10^{-6}, q=0.018, t=5.8$ ) (Fig. 4c). The effect of the MHC polygenic score on cg03805787 methylation was not mediated by the methylation of the CpGs in the MHC region. Additionally, this effect was not explained only by the HLA$D R B 1^{*} 1501$ genotype, as the effect of the MHC polygenic score remained significant $(p=0.002)$ after accounting for the effect of HLA-DRB1*1501 in the model. Replication analysis for cg03805787 could not be performed using data from the BLUEPRINT study, as measurement of cg03805787 methylation is not included in the Illumina $450 \mathrm{~K}$ array. However, we did replicate the association of the MHC polygenic score and cg03805787 methylation in $\mathrm{CD} 4^{+} \mathrm{T}$ cells from healthy participants in the PhenoGenetic study $(p=0.025$, Fig. 4c). No significant difference was found in cg03805787 methylation levels between primary and ex vivo-activated $\mathrm{CD} 4{ }^{+} \mathrm{T}$ cells in these 


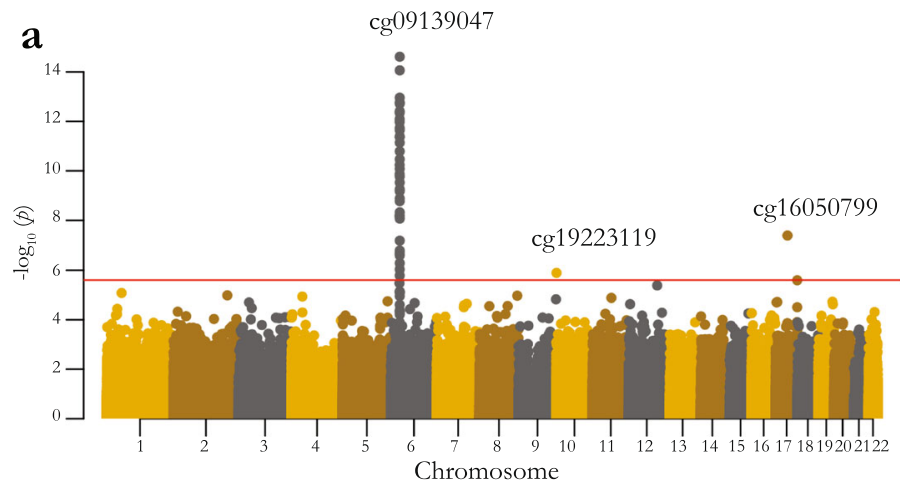

b
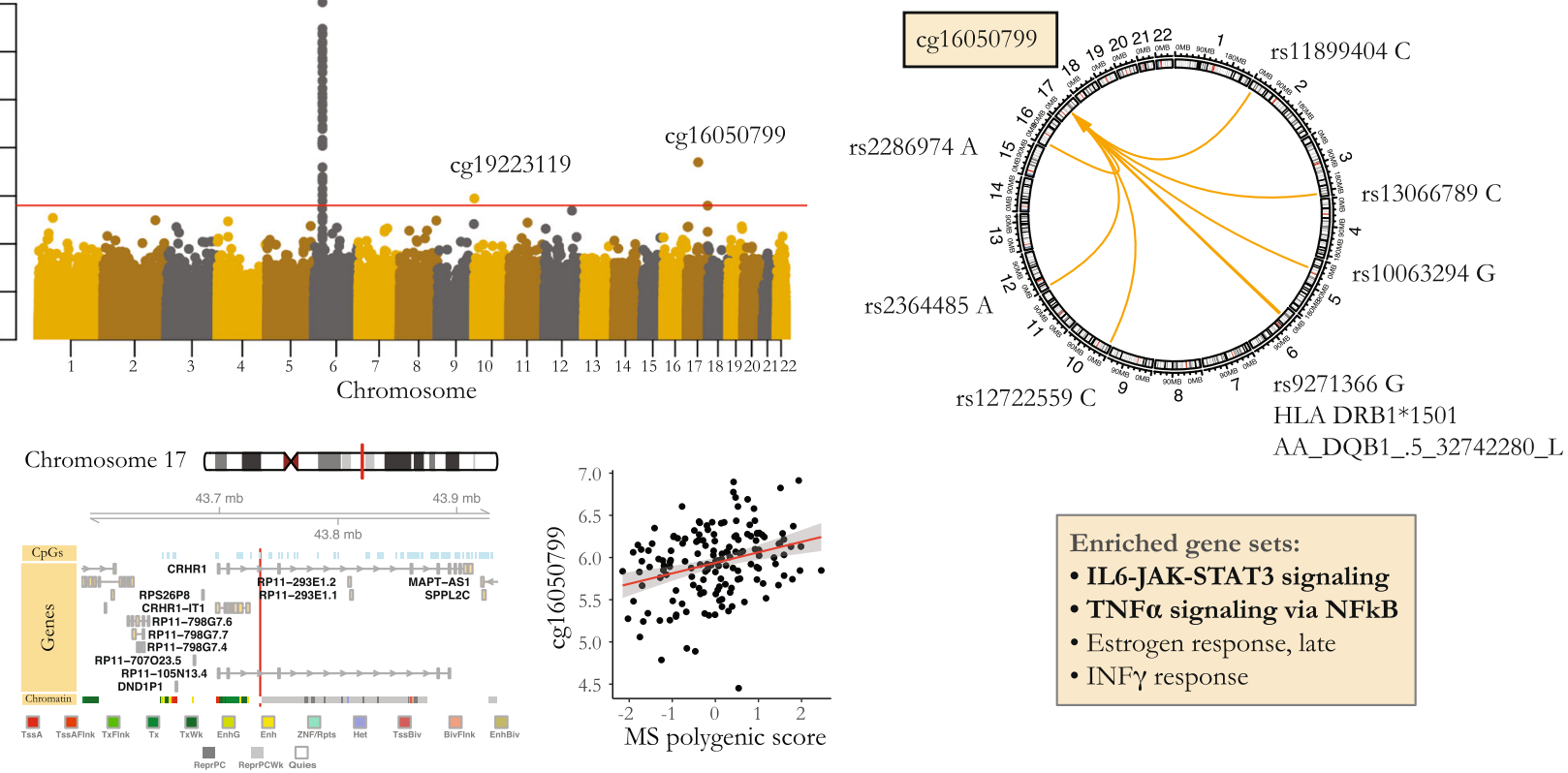

Enriched gene sets:

- IL6-JAK-STAT3 signaling

- TNF $\alpha$ signaling via NFkB

- Estrogen response, late

- INF $\gamma$ response

Fig. 5 DNA methylation effects of MS total polygenic score. a Manhattan plot shows association $p$-values between CpG methylation levels and MS total polygenic score. Red horizontal line shows the significance threshold (FDR $<5 \%$ ). Top CpGs in the three significant regions are specified. The genomic location for cg16050799 (the top associated CpG outside the MHC region, red vertical line) in relation to nearby CpGs, genes, and modeled chromatin states, in addition to its association with the MS total polygenic score (Z-scores) is further shown. Error band represents $95 \%$ confidence interval. Methylation levels are shown in M values. b Genomic locations of MS susceptibility alleles nominally associated with cg16050799 methylation ( $p<0.05$ ) are shown. Gene sets enriched for genes associated with these loci are specified.

individuals $(p=0.84)$. This finding on the effects of MHC genetic variation on DNA methylation in the PRKCA region opens an interesting arena for further investigation, as this gene may play a role as a focal point for the functional consequences of $\mathrm{MHC}$ variants.

DNA methylation effects of MS susceptibility total polygenic score. In our final analysis, we investigated the effects of two MS multi-locus polygenic scores on DNA methylation in order to find the targets for the aggregate effects of known MS susceptibility loci that could act as part of singular biological pathways. Our MWAS on the aggregate effects of non-MHC MS susceptibility loci (nonMHC polygenic score) on DNA methylation in CD $4^{+} \mathrm{T}$ cells did not result in any significant finding (all FDR-adjusted $p>0.05$ ). However, $38 \mathrm{CpGs}$ showed significant association with the MS total polygenic score (i.e., the sum of the MS MHC and non-MHC polygenic scores) at FDR $<0.05$ (Fig. 5a, Supplementary Data 5). 36 of these CpGs were located in the MHC and were also identified in association with the MS MHC polygenic score alone. The nonMHC polygenic score did not contribute to these associations. In contrast, the association of $2 \mathrm{CpGs}$ that were found outside of the MHC, on chromosomes 10 and 17, could not be explained by cis effects. Methylation of cg16050799 (in an intron of the CRHR1 gene on chromosome 17, Fig. 5a) was affected by independent and additive effects of both MHC $\left(p=7.6 \times 10^{-4}\right)$ and non-MHC $\left(p=1.9 \times 10^{-6}\right)$ polygenic scores, suggesting a convergence of the effects of multiple MS-associated loci on this methylation site. On the other hand, cg19223119 methylation (located in the DIP2C gene on chromosome 10) was mainly associated with the MHC polygenic score ( $p$ for MHC Score $=6.5 \times 10^{-6}, p$ for non-MHC Score $=0.024)$.

As cg16050799 methylation was affected by both MHC and non-MHC polygenic scores, we decided to further characterize this effect and identify the individual MS-associated loci which were driving this association. Although no individual association passed the multiple comparison testing correction (FDR $<0.05)$, results revealed suggestive $(p<0.05)$ associations between three MHC and six non-MHC risk loci and higher cg16050799 methylation. Gene set enrichment analysis performed on genes associated with the six non-MHC risk loci $(n=168$; details can be found in the "Methods") showed enrichment for the IL-6 and NF- $\mathrm{BB}$ pathways (Fig. 5b, Supplementary Data 6). Replication analysis could not be performed for cg16050799, as the methylation measurement was not available as part of the BLUEPRINT methylation array and the measurements performed in the PhenoGenetic samples did not pass quality control. In addition, the MHC variants were not imputed in the UC Berkeley study, and the association between non-MHC polygenic score and cg16050799 methylation was not significant in their whole blood sample. Given the shortcomings of our replication attempts, we could not determine the replicability of the observed association. However, we believe that convergent effect of both MHC and non-MHC polygenic scores on cg16050799 methylation in $\mathrm{CD}^{+} \mathrm{T}$ cells of MS patients deserves more thorough investigation in future studies.

\section{Discussion}

Our examination of the genetic architecture of DNA methylation profiles of primary CD4 ${ }^{+} \mathrm{T}$ cells from MS patients yielded two major products. First, we provide a new resource: a genome-wide cis-mQTL map for primary CD4 ${ }^{+} \mathrm{T}$ cells which is more extensive than the existing map (e.g., the cis-mQTLs available from the BLUEPRINT Epigenome Project ${ }^{9}$ ) due to a greater number of DNA methylation sites being assessed. Our extended T cell DNA methylation mQTL map will therefore be useful to a wide variety of investigators, including those outside of the field of MS. Results for the significant cis-mCpGs are available in Supplementary Data 1. Importantly, there is high concordance of the cis-mQTL 
effects in $\mathrm{CD} 4^{+} \mathrm{T}$ cells from MS patients (our study) with those found from healthy individuals (the BLUEPRINT and GOLDN studies), which (1) increases our confidence in the robustness of all resources and (2) suggests that the majority of our cis-mQTL effects are not specific to MS. This makes our cis-mQTL map an excellent exploratory resource for the study of cis functional effects of genetic loci associated with other diseases that have $\mathrm{CD}^{+} \mathrm{T}$ cell involvement and other $\mathrm{CD} 4^{+} \mathrm{T}$ cell-related traits such as $\mathrm{CD}^{+} \mathrm{T}$ cell functional phenotypes.

Second, in a series of analyses focused on MS, we identified not only the cis effects of individual MS susceptibility loci but also robust evidence for trans effects: (a) the MS locus near TBX6 on chromosome 16 influences methylation of a CpG island in the PRDM8 gene on chromosome 4, and (b) the MHC polygenic score affects methylation of an enhancer of PRKCA on chromosome 17. Both of these results are supported by replication analyses performed using independent datasets of healthy subjects and MS patients. Such robust trans-QTL effects are notoriously difficult to identify, and, here, they suggest that there may be convergence of the effects of multiple MS risk variants on DNA methylation of sites that are not themselves genetically implicated in MS.

PRDM8 belongs to a family of histone methyltransferases that are believed to act as negative regulators of transcription and play an important role in the development and cell differentiation. Hypermethylation of PRDM8 is associated with the first step of thymocyte differentiation (transition from thymic progenitors with lymphomyeloid potential to T-lineage-restricted progenitors) ${ }^{16}$. Among $\mathrm{T}$ cell subtypes, PRDM8 has higher expression levels in regulatory, memory and ex vivo activated $\mathrm{CD}^{+} \mathrm{T}$ cells ${ }^{6}$. Its chromatin accessibility increases upon stimulation in naïve $\mathrm{CD} 4^{+} \mathrm{T}$ cells, and the region remains accessible in memory $\mathrm{CD} 4^{+} \mathrm{T}$ cells ${ }^{17}$. Although PRDM8 has not previously been associated with MS, our replicated trans-mQTL findings suggests a role for this gene in the peripheral immune pathophysiology of MS as a downstream effect of the rs3809627 MS susceptibility variant.

Given that the MHC is by far the largest genetic risk factor for MS, the PRKCA region may offer a potential alternative to functional dissection of the genetically complex MHC region as we develop prevention strategies. The PRKCA locus has been linked genetically with MS in familial MS cases in independent sets of British ${ }^{18}$, Finnish and Canadian families ${ }^{19}$. Protein kinase $\mathrm{C}$ alpha ( $\mathrm{PKCa}$, encoded by the PRKCA gene) is a member of a family of serine/threonine protein kinases which is expressed ubiquitously in T cells and is involved in T cell receptor comodulation (i.e., downregulation of non-engaged $\mathrm{T}$ cell receptors following peptide-MHC complex engagement and $\mathrm{T}$ cell activation $)^{20}$. Programmed downregulation of $\mathrm{T}$ cell receptors is believed to represent a negative feedback mechanism that constrains $\mathrm{T}$ cell effector function in order to avoid excess inflammatory damage ${ }^{21}$. In addition, PKCa has been implicated in signaling pathways necessary for $\mathrm{T}$ cell interferon gamma ${ }^{22}$, interleukin (IL)-2 ${ }^{23}$ and IL-17 $\mathrm{A}^{24}$ production which are implicated in MS pathogenesis, and Prkca deficiency protects mice from experimental autoimmune encephalomyelitis (EAE, an MS animal model $)^{24}$. Finally, pharmacologic inhibition of PKCa has been shown to be beneficial in models of a number of other $\mathrm{T}$ cell-related immune conditions ${ }^{25}$. This extensive biological understanding of PRKCA is consistent with our observation that the effects of the MHC polygenic score on MS susceptibility may be exerted, in part, through this locus.

Likewise, our finding on the effect of MS total polygenic score on methylation of the CRHR1 region, if replicated, would provide an interesting target for modulation of the convergent effects of several MS susceptibility loci. CRHR1 is a receptor protein for corticotropin-releasing hormone (CRH). Peripheral CRH is believed to have proinflammatory effects, with $\mathrm{CRH}$-deficient mice showing an attenuated form of EAE and their $\mathrm{CD} 4^{+} \mathrm{T}$ cells showing reduced proliferation and a shift towards Th2 cytokine profile ${ }^{26}$. Hence, our finding might relate to the role of corticotropin hormone-related pathways in MS treatment or prevention.

In terms of the 19 MS susceptibility loci with colocalized cismQTL effects (with the TBX6 locus also having the colocalized trans-mQTL effect), seven were confirmed in the BLUEPRINT data, and the other 12, while significant in our analysis, will need further validation. While these analyses prioritize certain haplotypes as likely having effects on both MS susceptibility and cis or trans-DNA methylation, colocalization methods are limited in their ability to distinguish between two colocalized effects and two independent effects whose causal variants are in very high LD. Moreover, colocalization does not necessarily represent a mediatory causal relationship whereby one of the traits (e.g., DNA methylation) mediates the effect of genetic variation on the other trait (e.g., MS risk). A pleiotropic effect (i.e., independent effects of genetic variation on DNA methylation and MS risk) is alternatively possible, and the exact nature of the relationship (mediation, partial mediation, or full independence) cannot be resolved without large-scale longitudinal data. Nevertheless, colocalized MS and mQTL effects are reasonable candidates for further molecular and functional interrogation.

In addition, we acknowledge that our DNA methylation measurements were performed only on primary $\mathrm{CD} 4^{+} \mathrm{T}$ cells and not in the other cell types known to be involved in MS pathophysiology (such as B cells, NK cells, monocytes and microglia). Moreover, due to the cross-sectional design of our study, we were not able to take into account the dynamics of DNA methylation. Finally, our analyses are restricted to those CpGs interrogated by the array.

Although available information from previous eQTL and protein QTL studies have helped to identify the functional consequences of a number of MS risk variants ${ }^{2-5,27}$, it is notable that not all of our identified DNA methylation-associated MS variants have previously been associated with gene or isoform expression changes (Supplementary Data 3). This lack of gene-level information can stem from a number of reasons, including cell type- or context-specificity of the effects, or exclusion of genes with lower levels of total mRNA or isoform expression from prior analyses. In addition to providing complementary information to eQTL results, mQTL studies can be used to prioritize genetic loci for more thorough investigation of their gene-level effects. In line with this, and despite the moderate sample size, we were able to show that the rs7731626 MS variant's effect on ANKRD55 RNA expression is likely caused by altered DNA methylation in cis.

Our new data resource has therefore generated clear paths for further investigating the molecular events that contribute to the onset of MS at 19 of the more than 150 validated susceptibility loci. By sampling $\mathrm{CD}^{+} \mathrm{T}$ cells in an inflammatory disease population, we have not only validated the robustness of the previous MQTL results (BLUEPRINT and GOLDN studies) for this cell population, but also made the important observation that most of the evaluated mQTLs are not significantly different in health and disease. This makes our expanded set of 107,922 $\left(82,374\right.$ new) $\mathrm{CD}^{+} \mathrm{T}$ cell mQTLs of interest to a broad array of investigators interested in this important cell type outside of the MS context. Finally, our strategy of targeted trans-mQTL investigations returned replicable results in the PRDM8 and PRKCA loci, providing important evidence of the propagation of the functional consequences of MS variants outside of their immediate vicinity and a demonstration of the utility of our approach to find distal functional consequences. Our results will therefore help to ground future study designs to continue to elaborate the cascade of events leading to MS onset. 


\section{Methods}

\section{Main methylation study}

Subjects. Subjects were participants in the Comprehensive Longitudinal Investigation of Multiple Sclerosis at the Brigham and Women's Hospital (CLIMB) study ${ }^{7}$. CLIMB is a natural history observational study of MS, in which participants undergo semi-annual neurological examinations and annual magnetic resonance imaging and blood draw, from which PBMC are cryopreserved. In 2015, cryopreserved PBMC samples from subjects meeting the following criteria were pulled from the archive for sample processing: (1) age 18-55 years old, (2) a diagnosis of MS fulfilling 2010 McDonald criteria, (3) a relapsing-remitting disease course at the time of sampling, (4) being on disease-modifying therapies (either GA or DMF) at the time of sampling, (5) no evidence of disease activity in the prior 6 months, (6) no steroid use in the preceding 30 days, and (7) an EDSS score between 0 and 4 at the time of sampling. Of note, the patients were recruited at the time of clinical remission in order to avoid the transient and heterogeneous effects associated with clinical relapse and its treatment, while capturing the underlying chronic disease processes. The study was approved by the Institutional Review Board of the Brigham and Women's Hospital, and all participants had signed a written informed consent to participate in CLIMB. Samples from 208 patients who fulfilled the criteria were used for further processing.

Peripheral blood $\mathrm{CD}^{+} \mathrm{T}$ cell isolation. PBMC were collected prospectively from participants using the Immune Tolerance Network (https://www.immunetolera nce.org/) protocol to ensure data quality and to minimize variation among samples. In short, fresh blood was processed within $4 \mathrm{~h}$ using the Ficoll procedure to extract PBMC. The PBMC were then resuspended in fetal bovine serum with $10 \%$ DMSO and cryopreserved in liquid nitrogen. The samples meeting study criteria were removed from the sample archive and thawed in batches. Cell viability was accessed by acridine orange/propidium iodine staining and $\mathrm{CD} 4^{+} \mathrm{T}$ cells were isolated using a positive selection strategy implemented with Miltenyi magnetic CD4 MicroBeads (130-045-101). 1.7 million CD4 ${ }^{+} \mathrm{T}$ cells were obtained from each vial (range: 0.26-6.9 million). Isolated cells were resuspended into lysis buffer, and DNA was extracted from each sample using QIAamp DNA Blood Mini Kits (Qiagen 51104).

DNA methylation data. Whole-genome DNA methylation measurement was performed on DNA from purified CD4 ${ }^{+} \mathrm{T}$ cells using Infinium MethylationEPIC BeadChip (Illumina $850 \mathrm{~K}$ array) by the Center for Applied Genomics Genotyping Laboratory at the Children's Hospital of Pennsylvania. Quality control was performed using $\operatorname{minfi}{ }^{28}$ by comparing $\log 2$ median intensities of methylated and unmethylated channels, inspecting samples' beta distributions and control probes bisulfite conversion rates, and removing samples with detection $p$ values $>0.05$ at $>1 \%$ of the methylation sites. No sample was excluded at this step and average probe detection $p$ values were $<0.01$ for all samples. Further processing included normalization using Noob $^{29}$ and BMIQ ${ }^{30}$ algorithms (from minfi ${ }^{28}$ and

wateRmelan ${ }^{31}$ packages, respectively), removing methylation sites with beadcount $<3$ in $>5 \%$ of samples or detection $p$ value $>0.01$ in any sample, in addition to sites located on sex chromosomes, sites associated with probes with polymorphic targets with minor allele frequency $>1 \%$ in individuals with European ancestry ${ }^{32}$, and sites associated with cross-reactive probes ${ }^{32}$. M-values were calculated for 769,699 methylation sites to be used in statistical analysis. Data from probes measuring single nucleotide polymorphisms were also extracted to be used for identity check by cross-referencing with the genetic data.

Genotyping data. Whole-genome genotyping data were available for the majority of participants $(n=180)$ as part of previously genotyped batches of CLIMB samples using Illumina MEGA-EX and Affymetrix 6.0 arrays. Preprocessing of the genetic data was performed using PLINK $2.0^{33}$. SNPs with call rate $<95 \%$ and samples with genotyping rate $<90 \%$, mismatch between recorded sex and genetic sex, and low or high heterozygosity rates ( $>3$ standard deviation) were removed. Data from the two genotyping batches were merged and samples from related individuals (pi-hat $>$ 0.125 ) were also excluded. Multidimensional scaling was performed using HapMap3 reference data. Samples from individuals of non-European ancestry and ethnic outliers with $>3$ standard deviation difference from the European samples were identified and further removed (final $N$ at this stage $=158$ ). Imputation was performed for each genotyping batch separately using the Michigan Imputation Server $^{34}$ and the Haplotype Reference Consortium (HRC) panel v1.1. Imputed data from the study participants were merged, and SNPs with low imputation quality $\left(R^{2}<0.8\right)$ were removed.

Final dataset. Principal component (PC) analysis was performed on the methylation data from individuals that passed genetic data quality control $(n=158)$. The first four principal components explained 39\% of the total variability (Supplementary Fig. 11) and were used to remove outlier samples from the methylation data ( $>3$ standard deviation on any of the 4 PC values). Two additional samples were removed using this measure, and the final dataset consisted of data from 156 individuals (demographic details are available in Table 1).

$m Q T L$ analyses (cis and trans). We used QTLtools ${ }^{35}$ to perform genome-wide mapping of $\mathrm{CD}^{+} \mathrm{T}$ cell cis-mQTLs, as well as targeted mapping of trans-mQTLs among SNPs in $100 \mathrm{~kb}$ of the lead autosomal non-MHC MS-associated SNPs reported in ${ }^{2}$. SNPs with minor allele frequency $<0.05$, minor allele count $<15$, and departure from Hardy-Weinberg equilibrium $\left(p<1 \times 10^{-6}\right)$ were excluded from mQTL analyses.

For the cis-mQTL analysis, linear regression analysis was performed between quantile normalized DNA methylation M-values and imputed genotype dosages for SNPs in cis $( \pm 1 \mathrm{Mb})$ of each CpG site, accounting for the effects of age, sex, treatment, genotyping array, the first three genotyping PCs, and the first FDR methylation PCs. The nominal $p$ values for the top variants in $c i s$ were then adjusted for the number of tests performed in cis using a permutation scheme $(n=1000$ permutations) which models the null distribution of associations using a beta distribution. Finally, to account for the multiple $\mathrm{CpG}$ sites tested across the whole genome, significant associations were reported at 5\% False Discovery Rate (FDR).

For the trans-mQTL analysis, linear regression analysis was performed between SNPs in $\pm 100 \mathrm{~kb}$ of the lead MS-associated SNPs and all CpG sites outside their cis windows, accounting for the effects of the previously mentioned covariates. The nominal $p$-values were adjusted for the number of variants being tested using the null distribution of associations built from beta approximation of permutation outcome by permuting and testing associations between randomly selected 10,000 $\mathrm{CpG}$ methylation levels and all included variants. FDR adjustment was then implemented to account for the multiple CpG sites being tested.

Colocalization analyses. Analyses were performed using the $\mathrm{R}$ coloc package ${ }^{12}$ in order to determine whether MQTL effects and MS susceptibility effects that are located in close proximity of each other are likely the result of a shared causal variant. Briefly, for each autosomal non-MHC MS locus, mQTL effects whose lead SNPs were located in $100 \mathrm{~kb}$ of the lead MS SNP were identified. Colocalization of the effects of each identified MS-mQTL pair was assessed separately. The input data for each analysis consisted of: 1) summary association statistics ( $p$ value, beta -regression coefficient-, variance of beta, sample size, and minor allele frequency) of SNPs in $100 \mathrm{~kb}$ of the lead MS SNP extracted from the discovery phase of the IMSGC GWAS study $^{2}$ ( $n=14,802 \mathrm{MS}$ and 26,703 control participants), as well as our cis or trans mQTL analyses; 2) the suggested prior probabilities ${ }^{12}$ of $1 \times 10^{-4}$ for the association between each SNP and each of the two traits, and $1 \times 10^{-6}$ for the association between each SNP and both traits. The posterior probability of each locus containing a causal variant affecting both MS and mQTL effects was estimated against the probabilities of other models (a null model of no association, association with only the first or the second trait, or independent associations with each of the two traits). Results are reported at a posterior probability cut off of 0.8 for the colocalized effects.

Polygenic score calculations. MS polygenic scores were calculated based on the genome-wide significant results from the 2019 IMSGC MS GWAS study (31 MHC markers and 200 autosomal non-MHC SNPs) ${ }^{2}$. SNPs, amino acids, and HLA alleles in the MHC region were imputed using the SNP2HLA package ${ }^{36}$ and the Type 1 Diabetes Genetics Consortium HLA reference panel. The MS MHC polygenic scores were then calculated as the sum of the imputed dosages of the $31 \mathrm{MHC}$ markers associated with MS multiplied by their effect sizes ${ }^{2}$ (log odds ratio). For the MS total polygenic score, SNP dosages for the autosomal non-MHC MS SNPs (or if not available, tag SNPs in LD $>0.8$ ) were extracted from the whole-genome imputed data (imputed using the Michigan Imputation Server ${ }^{34}$ and the HRC reference panel), multiplied by their effect sizes, and added to the MHC polygenic score. Data from 10 of the 200 non-MHC SNPs were not available in the qualitycontrolled imputed data and were substituted by a close by SNP in LD $>0.8$. Data from seven SNPs were not included in the MS total polygenic score, as no data were available for them or SNPs in $\mathrm{LD}>0.8$ with them. The list of MHC and nonMHC markers used in the calculation of the MS total polygenic score and their effect sizes can be found in Supplementary Data 7.

Methylome-wide association studies. GLINT ${ }^{37}$ was used to perform the methylomewide association studies (MWAS). The final methylation dataset was used to assess the associations between DNA methylation and MS polygenic scores at each of the methylation sites, while adjusting for the effects of age, sex, treatment, genotyping batch, the first three genotyping PCs and the first four methylation PCs. Significant results were reported at $5 \% \mathrm{FDR}$.

Gene mapping and gene set enrichment analysis. We used FUMA ${ }^{38}$ (v1.3.3d) SNP2GENE function for gene mapping and GENE2FUNC function for gene set enrichment analysis. Briefly, genotypes were mapped to genes (Ensembl Release 92) using the following parameters: Input genotypes were SNPs in LD with the lead SNPs ( $\mathrm{r} 2>0.6)$; $p$ value $<0.05$; reference panel population 1000G Phase3 EUR; minor allele frequency $>0.01$; merging distance between LD blocks $<250 \mathrm{~kb}$. Gene mapping was performed by positional, eQTL and 3D chromatin interaction mapping using all available annotation datasets and default parameters (positional mapping window: $100 \mathrm{~kb}$, eQTL: FDR $<0.05$, chromatin interaction: $\mathrm{FDR}<1 \mathrm{e}-6$, promoter region window $250 \mathrm{bp}$ upstream and $500 \mathrm{bp}$ downstream of transcription start sites). The mapped genes were then fed to the GENE2FUNC function for pathway analysis. Minimum overlap with each gene set was set to two genes. Results were reported at 5\% FDR.

Statistical analysis. Post-hoc linear regression studies were performed while accounting for the effects of age, sex, treatment, genotyping batch, the first three 
genotyping PCs, and the first four methylation PCs, unless otherwise specified. Enrichment analyses for chromatin states were performed using Chi-square tests. Mediation analyses for assessing the significance of the direct and causal mediated effects between genetic variation, DNA methylation, and RNA expression were performed using $\mathrm{R}$ mediation package ${ }^{39}$. All reported $p$-values are two-sided. All results are reported in GRCh37/hg19 genomic coordinates.

RNA sequencing sub-study. RNA sequencing data from peripheral blood purified naïve and memory $\mathrm{CD} 4^{+} \mathrm{T}$ cells were available from a subset of participants of the main DNA methylation study as part of a separate and larger RNA sequencing project ( $n=42$ and 43 naïve and memory $\mathrm{CD} 4^{+} \mathrm{T}$ cell samples, respectively). Data from participants who had changed medication between the sampling dates for DNA methylation and RNA sequencing studies were excluded ( $n=6$ from each cell type). All remaining RNA sequencing samples were from patients receiving glatiramer acetate at the time of sampling for both studies. All RNA sequencing samples were taken within 5 years of the sampling date for the DNA methylation study, with $80 \%$ being performed within one year of the DNA methylation sampling. PBMC sampling and $\mathrm{CD} 4^{+} \mathrm{T}$ cells isolation were performed using the same protocol used in the DNA methylation study. Naïve and memory $\mathrm{CD} 4^{+} \mathrm{T}$ cells were isolated on a BD FACSAria flow cytometer using fluorochrome-labeled antiCD3 (1:50 dilution, $2 \mu \mathrm{l}$ to $100 \mu \mathrm{l}$ staining volume containing $1 \times 10^{6}$ cells), antiCD4 (1:50 dilution), and anti-CD45RA (1:33 dilution) antibodies. Whole transcriptome 25-bp paired end sequencing was performed using the Broad Institute HiSeq 2500 platform to an average depth of 15 million reads. Processing was performed according to the Broad Institute RNA-seq pipeline for the GTEx Consortium. Briefly, RNA sequence reads were aligned to the GRCh38/hg38 genome reference using $\mathrm{STAR}^{40}$, quality control was performed using RNA$\mathrm{SeQC}^{41}$, and quantification of gene expression levels was performed using $\mathrm{RSEM}^{42}$ Transcripts with low expression values (average TPM $<2$ ) were removed. Samples with outlier average correlation with the other samples $\left(\mid D\right.$ statistic $^{43} \mid>3$ standard deviation from the mean) were excluded from further analysis (one memory $\mathrm{CD} 4^{+}$ $\mathrm{T}$ cell sample was removed). TPM values were log-transformed and quantilenormalized. Gene start and end positions were extracted from Ensembl Release 93 annotations. Linear regression modeling was used to investigate the associations between methylation levels of CpGs of interest-identified from cis, trans, and polygenic score analyses-and mRNA expression levels of genes within $\pm 1 \mathrm{Mb}$ of their respected CpGs, while adjusting for the effects of age and sex. In total, 912 association analyses were performed for naïve $\mathrm{CD} 4^{+} \mathrm{T}$ cells assessing associations between 49 CpGs and 488 genes, and 887 association analyses were performed for memory $\mathrm{CD} 4^{+} \mathrm{T}$ cells between $49 \mathrm{CpGs}$ and 480 genes, each using data from 36 participants. Associations with FDR-adjusted $p<0.05$ were considered significant.

\section{Additional datasets}

BLUEPRINT study. BLUEPRINT Epigenome Project's publicly available cis-mQTL summary statistics ${ }^{9}$ from the study of naïve $\mathrm{CD} 4^{+} \mathrm{T}$ cells were used for comparison with our cis-mQTL and colocalization findings. DNA methylation measurements were performed using Illumina Infinium HumanMethylation450 BeadChips, and samples were from 132 healthy individuals of European origin (Table 1). In order to perform replication analysis for our trans-mQTL and polygenic score analyses findings, we downloaded the BLUEPRINT genetic data from the European Genome-phenome Archive and performed whole-genome and MHC imputation of the data in a similar manner to the main methylation study. Associations were performed between genetic measures and BLUEPRINT's publicly available processed DNA methylation data ( $M$ values), accounting for the effects of age and sex.

UC Berkeley study. Data from this study were both gathered and analyzed independently by the UC Berkeley investigators. Whole blood samples from 208 selfidentified white, female MS patients were used for the study (Table 1). Genomewide DNA methylation was profiled using Infinium MethylationEPIC BeadChips. Methylation data were analyzed using Bioconductor minfi package ${ }^{28}$. Background dye correction and quantile normalization were performed, followed by the removal of batch effects using the ComBat ${ }^{44}$ function of $\mathrm{R}$ sva package. Ancestry and cell-type heterogeneity were estimated using GLINT ${ }^{37}$, and methylation M values were adjusted for ancestry and cell type components as well as for age at sampling. Genome-wide genotyping was performed using Illumina Infinium $660 \mathrm{~K}$ OmniExpress or OmniExpressExome BeadChip arrays. Merged $660 \mathrm{~K}$ and Omni Express genotyping dataset (273,906 SNPs) were phased using SHAPEIT2, and imputed against reference haplotypes from Phase 3 of the 1000 Genomes Project using IMPUTE4. Linear regression models were used to study the association between adjusted M values and genotypes, while covarying for MS treatment status (0/1, never/ever treated). MHC imputation was not available for this study.

PhenoGenetic study. Frozen PBMC samples from 48 healthy participants of European origin from the PhenoGenetic Project who were part of the ImmVar study $^{3,13}$ and had available genotyping data were selected for replication analyses (Table 1). $\mathrm{CD} 4^{+} \mathrm{T}$ cells were purified from cryopreserved PBMC using a magnetic microbead-based strategy (Miltenyi 130-096-533) after thawing with $10 \mathrm{ml}$ PBS. Cells from a subset of the samples $(n=28)$ were split in half, and one half was placed in culture with serum-free X-Vivo medium and then stimulated using
$2 \mu \mathrm{g} / \mathrm{ml}$ anti-CD3 and $1 \mu \mathrm{g} / \mathrm{ml}$ anti-CD28 antibodies. Cells were collected $24 \mathrm{~h}$ after stimulation. DNA was extracted from the 48 primary and 28 in vitro activated $\mathrm{CD}^{+} \mathrm{T}$ cell samples and underwent bisulfite conversion. Methylation levels of CpGs of interest were measured using the Agena Bioscience mass spectrometrybased EpiTYPER assay, with primers designed using EpiDesigner (Supplementary Data 8). Genotyping was performed as part of two larger batches of samples previously genotyped using Illumina Infinium OmniExpressExome and Illumina MEGA-EX arrays. Whole-genome and MHC imputation were performed as mentioned in the main methylation study. Association analyses were performed between genetic measures and DNA methylation $M$ values, adjusting for the effects of age and sex. Paired $t$ tests were used to compare the methylation levels between primary and activated cells at each $\mathrm{CpG}$ site.

GOLDN study. Available baseline data from 717 related participants from the Genetics of Lipid-lowering Drugs and Diet Network (GOLDN) study (dbGaP Study Accession: phs000741.v2.p1) were used for the cis-mQTL replication analyses (Table 1). $\mathrm{CD} 4^{+} \mathrm{T}$ cells were isolated from peripheral blood at the time of fasting. Genotyping was performed using Affymetrix 6.0 array, and imputation was performed using $\mathrm{MaCH}^{45}$ with HapMap Phase II (release 22, Human Genome build 36) as the reference. Genome-wide DNA methylation was profiled using Illumina Infinium HumanMethylation450 BeadChips, and beta scores were derived using Illumina's GenomeStudio software. QC details can be found at ${ }^{46}$. Association analyses were performed between genotypes and DNA methylation beta values in two steps: 1) adjusting the methylation beta values for the fixed effects of methylation plate ID and methylation array position and the random effect of methylation array ID;2) association of the adjusted beta-values with genotypes while accounting for the effects of age, sex, study center, the first four DNA methylation PCs, and kinship (modeled using $\operatorname{lmekin}^{47}$ function of R kinship package).

Reporting summary. Further information on research design is available in the Nature Research Reporting Summary linked to this article.

\section{Data availability}

The genotyping and DNA methylation data from our MS CD4 ${ }^{+} \mathrm{T}$ cell dataset have been deposited in the Synapse database under accession codes syn26340457 [https://doi.org/ 10.7303/syn26340457] and syn26339303 [https://doi.org/10.7303/syn26339303], respectively. As sensitive human data, these data can be accessed upon request, following the establishment of a Data Use Agreement with the Brigham and Women's Hospital. The initial request can be sent to the corresponding author, and the applicants will be contacted within a week. The complete set of summary statistics from the cis-mQTL analysis are available for download from the Synapse database under accession code syn26339302 [https://doi.org/10.7303/syn26339302]. The genome-wide significant cismQTL effects can be found in Supplementary Data 1. Data from the Genetics of Lipidlowering Drugs and Diet Network (GOLDN) study used for the cis-mQTL replication analyses can be obtained from dbGaP under accession code phs000741.v2.p1 [https:// www.ncbi.nlm.nih.gov/projects/gap/cgi-bin/study.cgi?study_id=phs000741.v2.p1]. BLUEPRINT Epigenome Project's ${ }^{9}$ publicly available cis-mQTL summary statistics were downloaded from ftp.ebi.ac.uk/pub/databases/blueprint/blueprint_Epivar/. BLUEPRINT genetic data were accessed from the European Genome-phenome Archive under accession code EGAD00001002663.

Received: 31 May 2020; Accepted: 18 November 2021; Published online: 06 December 2021

\section{References}

1. Thompson, A. J., Baranzini, S. E., Geurts, J., Hemmer, B. \& Ciccarelli, O. Multiple sclerosis. Lancet 391, 1622-1636 (2018).

2. International Multiple Sclerosis Genetics Consortium. Multiple sclerosis genomic map implicates peripheral immune cells and microglia in susceptibility. Science 365, eaav7188 (2019).

3. Raj, T. et al. Polarization of the effects of autoimmune and neurodegenerative risk alleles in leukocytes. Science 344, 519-523 (2014).

4. James, T. et al. Impact of genetic risk loci for multiple sclerosis on expression of proximal genes in patients. Hum. Mol. Genet. 27, 912-928 (2018).

5. Chun, S. et al. Limited statistical evidence for shared genetic effects of eQTL and autoimmune-disease-associated loci in three major immune-cell types. Nat. Genet. 49, 600-605 (2017).

6. Schmiedel, B. J. et al. Impact of genetic polymorphisms on human immune cell gene expression. Cell 175, 1701-1715.e16 (2018).

7. Gauthier, S. A., Glanz, B. I., Mandel, M. \& Weiner, H. L. A model for the comprehensive investigation of a chronic autoimmune disease: the multiple sclerosis CLIMB study. Autoimmun. Rev. 5, 532-536 (2006).

8. $\mathrm{Ng}$, B. et al. An xQTL map integrates the genetic architecture of the human brain's transcriptome and epigenome. Nat. Neurosci. 20, 1418-1426 (2017). 
9. Chen, L. et al. Genetic drivers of epigenetic and transcriptional variation in human immune cells. Cell 167, 1398-1414.e24 (2016).

10. Roadmap Epigenomics Consortium, A. et al. Integrative analysis of 111 reference human epigenomes. Nature 518, 317-330 (2015).

11. Maltby, V. E. et al. DNA methylation changes in CD4 $+\mathrm{T}$ cells isolated from multiple sclerosis patients on dimethyl fumarate. Mult. Scler. J. Exp. Transl. Clin. 4, 205521731878782 (2018).

12. Giambartolomei, C. et al. Bayesian test for colocalisation between pairs of genetic association studies using summary statistics. PLoS Genet. 10, e1004383 (2014).

13. De Jager, P. L. et al. ImmVar project: insights and design considerations for future studies of "healthy" immune variation. Semin. Immunol. 27, 51-57 (2015).

14. Kular, L. et al. DNA methylation as a mediator of HLA-DRB1*15:01 and a protective variant in multiple sclerosis. Nat. Commun. 9, 2397 (2018).

15. Maltby, V. E. et al. Differential methylation at MHC in CD4+ T cells is associated with multiple sclerosis independently of HLA-DRB1. Clin. Epigenet. 9, 71 (2017).

16. Rodriguez, R. M. et al. Regulation of the transcriptional program by DNA methylation during human $\alpha \beta$ T-cell development. Nucleic Acids Res. 43, 760-774 (2015).

17. $\mathrm{Tu}, \mathrm{W}$. J. et al. Priming of transcriptional memory responses via the chromatin accessibility landscape in T cells. Sci. Rep. 7, 44825 (2017).

18. Sawcer, S. et al. A genome screen in multiple sclerosis reveals susceptibility loci on chromosome 6p21 and 17q22. Nat. Genet. 13, 464-468 (1996).

19. Saarela, J. et al. PRKCA and multiple sclerosis: association in two independent populations. PLoS Genet. 2, e42-e42 (2006).

20. von Essen, $\mathrm{M}$. et al. Protein kinase C (PKC) alpha and PKC theta are the major PKC isotypes involved in TCR down-regulation. J. Immunol. 176, 7502-7510 (2006).

21. Gallegos, A. M. et al. Control of T cell antigen reactivity via programmed TCR downregulation. Nat. Immunol. 17, 379-386 (2016).

22. Pfeifhofer, C. et al. Defective IgG2a/2b class switching in PKC alpha-/- mice. J. Immunol. 176, 6004-6011 (2006).

23. Iwamoto, T. et al. Accelerated proliferation and interleukin-2 production of thymocytes by stimulation of soluble anti-CD3 monoclonal antibody in transgenic mice carrying a rabbit protein kinase C alpha. J. Biol. Chem. 267, 18644-18648 (1992).

24. Meisel, M. et al. The kinase PKCa selectively upregulates interleukin-17A during Th17 cell immune responses. Immunity 38, 41-52 (2013).

25. Haarberg, K. M. K. et al. Pharmacologic inhibition of PKCa and PKC $\theta$ prevents GVHD while preserving GVL activity in mice. Blood 122, 2500-2511 (2013).

26. Benou, C. et al. Corticotropin-releasing hormone contributes to the peripheral inflammatory response in experimental autoimmune encephalomyelitis. $J$. Immunol. 174, 5407-5413 (2005).

27. Matesanz, F. et al. A functional variant that affects exon-skipping and protein expression of SP140 as genetic mechanism predisposing to multiple sclerosis. Hum. Mol. Genet. 24, 5619-5627 (2015).

28. Aryee, M. J. et al. Minfi: a flexible and comprehensive Bioconductor package for the analysis of Infinium DNA methylation microarrays. Bioinformatics 30, 1363-1369 (2014).

29. Triche, T. J., Weisenberger, D. J., Van Den Berg, D., Laird, P. W. \& Siegmund, K. D. Low-level processing of Illumina Infinium DNA Methylation BeadArrays. Nucleic Acids Res. 41, e90 (2013).

30. Teschendorff, A. E. et al. A beta-mixture quantile normalization method for correcting probe design bias in Illumina Infinium $450 \mathrm{k}$ DNA methylation data. Bioinformatics 29, 189-196 (2013).

31. Pidsley, R. et al. A data-driven approach to preprocessing Illumina $450 \mathrm{~K}$ methylation array data. BMC Genom. 14, 1-10 (2013).

32. McCartney, D. L. et al. Identification of polymorphic and off-target probe binding sites on the Illumina Infinium MethylationEPIC BeadChip. Genomics Data 9, 22-24 (2016).

33. Chang, C. C. et al. Second-generation PLINK: rising to the challenge of larger and richer datasets. Gigascience 4, 7 (2015).

34. Das, S. et al. Next-generation genotype imputation service and methods. Nat. Genet. 48, 1284-1287 (2016)

35. Delaneau, O. et al. A complete tool set for molecular QTL discovery and analysis. Nat. Commun. 8, 15452 (2017).

36. Jia, X. et al. Imputing amino acid polymorphisms in human leukocyte antigens. PLoS ONE 8, e64683 (2013).

37. Rahmani, E. et al. GLINT: a user-friendly toolset for the analysis of highthroughput DNA-methylation array data. Bioinformatics 33, 1870-1872 (2017).

38. Watanabe, K., Taskesen, E., van Bochoven, A. \& Posthuma, D. Functional mapping and annotation of genetic associations with FUMA. Nat. Commun. 8, 1826 (2017).
39. Tingley, D., Yamamoto, H. T., Hirose, K., Keele, L. \& Princeton, K. I. mediation: R Package for Causal Mediation Analysis. http://cran.r-project.org/ package $=$ mediation.

40. Dobin, A. et al. STAR: ultrafast universal RNA-seq aligner. Bioinformatics 29, 15-21 (2013)

41. DeLuca, D. S. et al. RNA-SeQC: RNA-seq metrics for quality control and process optimization. Bioinformatics 28, 1530-1532 (2012).

42. Li, B. \& Dewey, C. N. RSEM: accurate transcript quantification from RNA-Seq data with or without a reference genome. BMC Bioinform. 12, 323 (2011).

43. GTEx Consortium, K. G. et al. Human genomics. The Genotype-Tissue Expression (GTEx) pilot analysis: multitissue gene regulation in humans. Science 348, 648-660 (2015)

44. Johnson, W. E., Li, C. \& Rabinovic, A. Adjusting batch effects in microarray expression data using empirical Bayes methods. Biostatistics 8, 118-127 (2007).

45. Li, Y., Willer, C. J., Ding, J., Scheet, P. \& Abecasis, G. R. MaCH: Using sequence and genotype data to estimate haplotypes and unobserved genotypes. Genet. Epidemiol. 34, 816-834 (2010).

46. Irvin, M. R. et al. Genomics of post-prandial lipidomic phenotypes in the Genetics of Lipid Lowering Drugs and Diet Network (GOLDN) study. PLoS ONE 9, e99509 (2014)

47. Therneau, T. The lmekin function. (2020).

\section{Acknowledgements}

We are grateful to all the participants for their invaluable contribution to the study. We also thank Albert Boulanger for helping with data organization for the purpose of sharing the summary statistics. The study was funded by a grant to PLD from the Massachusetts Life Sciences Center.

\section{Author contributions}

P.K., S.M.C., A.K., C.Y., B.J.K., and J.L. contributed to data acquisition. T.R., H.K., Y.M., D.F., X.S., and B.R. contributed to data analysis. T.R. and P.L.D. contributed to the analysis plan. P.L.D. and H.L.W. supervised the CLIMB study data acquisition. L.F.B. supervised the UC Berkeley study data acquisition and analysis. J.M.O., D.M.A., and D.K.A. supervised the GOLDN study data acquisition and analysis. P.L.D. supervised the PhenoGenetic study data acquisition and analysis. T.R. and P.L.D. contributed to the interpretation of results and drafted the manuscript. T.R., H.K., Y.M., D.F., P.K., S.M.C., A.K., C.Y., B.J.K., X.S., B.R., J.M.O., D.M.A., D.K.A., J.L., N.P., L.F.B., H.L.W., and P.L.D. revised the manuscript. P.L.D. supervised the whole work.

\section{Competing interests}

The authors declare no competing interests.

\section{Additional information}

Supplementary information The online version contains supplementary material available at https://doi.org/10.1038/s41467-021-27427-w.

Correspondence and requests for materials should be addressed to Philip L. De Jager.

Peer review information Nature Communications thanks Corey Watson and the other, anonymous, reviewer(s) for their contribution to the peer review of this work.

Reprints and permission information is available at http://www.nature.com/reprints

Publisher's note Springer Nature remains neutral with regard to jurisdictional claims in published maps and institutional affiliations.

Open Access This article is licensed under a Creative Commons Attribution 4.0 International License, which permits use, sharing, adaptation, distribution and reproduction in any medium or format, as long as you give appropriate credit to the original author(s) and the source, provide a link to the Creative Commons license, and indicate if changes were made. The images or other third party material in this article are included in the article's Creative Commons license, unless indicated otherwise in a credit line to the material. If material is not included in the article's Creative Commons license and your intended use is not permitted by statutory regulation or exceeds the permitted use, you will need to obtain permission directly from the copyright holder. To view a copy of this license, visit http://creativecommons.org/ licenses/by/4.0/

(c) The Author(s) 2021 